EUROPEAN JOURNAL OF PURE AND APPLIED MATHEMATICS

Vol. 15, No. 1, 2022, 314-327

ISSN 1307-5543 - ejpam.com

Published by New York Business Global

\title{
Numerical Study of Two Dimensional Steady Incompressible Newtonian Laminar Flow of Blood Through Constricted Artery
}

\author{
Jonathan Tsetimi ${ }^{1, *}$, Ebimene Mamadu ${ }^{1}$ \\ 1 Department of Mathematics, Faculty of Science, Delta State University, Abraka, \\ Delta State, Nigeria
}

\begin{abstract}
In this work, a numerical study of two dimensional steady, incompressible Newtonian laminar flow of blood through a stenosed (constricted) artery is investigated. The Variational Iteration Method (VIM) is employed to obtain the analytic expressions for the velocity profile and pressure gradient. The results obtained are graphically discussed for mild stenosis (25\%) for low Reynolds number in the range 20-100. The significance of the analysis is identified by comparing the results obtained with similar studies in existing literature, and established a good agreement. The results obtained reveal alterations in blood flow due to presence of stenosis in arterial wall and the implications are discussed.
\end{abstract}

2020 Mathematics Subject Classifications: 58J05, 65B10, 65K10, 76D05

Key Words and Phrases: Hemodynamic, Newtonian fluid, Cardiovascular disease, Atherosclerosis, Stenosis, Artery, Laminar flow

\section{Introduction}

The study of flow of blood through the human circulatory system has been a subject of attention for scientific research for about a couple of centuries. Like so many problems of nature including life sciences, hemodynamics is a rigorous one due to complex nature of blood, the circulatory system and their constituent elements. Hemodynamics is the study of blood mechanical and physiological characteristics and how it flows in the body, as well as the forces involved. It describes the physical laws that govern blood flow in the blood vessels. Proper blood circulation (blood flow) is a condition necessary for adequate supply of oxygen to all body tissues, which thereafter support cardiovascular health, longevity, high quality of life and survival of surgical patients. There are numerus factors influencing hemodynamics ranging from circulatory volume of blood, viscosity of blood, resistance, vascular diameter, respiration, density, obstruction and so on. There are

${ }^{*}$ Corresponding author.

DOI: https://doi.org/10.29020/nybg.ejpam.v15i1.4245

Email addresses: jtsetimi@delsu.edu.ng (J. Tsetimi), emamadu@delsu.edu.ng (E. J. Mamadu) 
various diseases and disorders of the cardiovascular system associated with hemodynamic dysfunction, due to mild or intense narrowing or blockage of arteries. Some disease of the cardiovascular system includes atherosclerosis, aneurysm, thrombosis, stenosis, etc. Majority of cardiovascular diseases and disorders are connected to systemic dysfunction of which heart failure, stroke and hypertension are prominent known ones. In this work, we shall focus on blood flow through an artery with presence of stenosis, and effect on flow properties such as velocity profile and pressure gradient.

Blood is a fluid which circulates through the hearts, arteries, veins and capillaries of the circulatory system. Blood is composed of two parts; a solid portion which is about $(40-45 \%)$ formed element, consisting of the Red blood cells (for oxygen and carbon dioxide transportation), the white blood cells (for defense and immunity) and Platelets (blood clothing) all suspended in plasma. The liquid portion (Plasma) makes up the majority $(45-60 \%)$ of the blood volume. Plasma is mainly made up of (about $90 \%$ ) of water. The density of blood is approximately $1060 \mathrm{~kg} / \mathrm{m}^{3}$ at $37^{0} \mathrm{C}$. Blood Plasma has a density of $1025 \mathrm{~kg} / \mathrm{m}^{3}$ which is predominantly about $93 \%$ by volume of water. The viscosity of a fluid describes the internal friction between fluid particles as they slide past one another. Generally, the viscosity of blood is a function of plasma viscosity (which is relatively constant). When the viscosity is constant, blood behaves like a Newtonian fluid since the blood cells do not deform. The viscosity of plasma is approximately $\mu=$ $3.2 \times 10^{-1} \mathrm{~kg} \cdot \mathrm{m}^{-1} \mathrm{~s}^{-1}[1]$.

The study of blood flow through the cardiovascular system has gained tremendous interest in scientific research. A number of theoretical and scientific efforts has been made in literature to explain the behavior of blood when it flows through the vessels of circulatory system of living beings. Several researchers such as [2], have reported that the fluid dynamics and rheological properties of blood and its flow could play an important role in the fundamental understanding, treatment and diagnosis of many cerebrovascular, cardiovascular and arterial diseases. Yamaguchi [3] gave an insight to the cardiovascular system, its physical nature, pathological role, measurements and analysis. Mark [5] have used the poiseuille's law and Bernoulli's equation in a flow tube with changing cross sectional area to conclude that pressure drops as velocity increases in the narrowed portion of the tube.

Several other investigators have presented some theoretical and numerical analysis to study blood flow characteristics due to the presence of stenosis in the arteries. Young [4] investigated the effects of a time-dependent stenosis on flow passing through a tube and analyzed the effects of stenosis on flow characteristics of blood by treating blood as a Newtonian fluid. The work of [6] also reports that, an increase in the size of stenosis increases both the wall shear stress and impedance. An approximate solution is presented in [5] to the problem of incompressible flow passing through an axisymmetric constriction. The study found theoretical results for velocity distribution, wall shearing stress, pressure drop and separation phenomena for both mild and severe stenosis for Reynolds numbers below transition. The results reveal significant alterations in flow caused by stenosis. A detailed description is given in [6] of the effect of varying degrees of stenosis on arterial pulsatile waveforms. Blood flow measurements were made using the electromagnetic blood flow 
meter proximal and distal to the stenosis. Kapur [7] gave a survey on some mathematical models for the problems - pulsatile blood flows in rigid and elastic tubes, flow of blood in arteries with stenosis etc.

Pralhad and Schultz [8] presented a blood flow model in a stenosed tube by representing blood flow by a couple stress fluid. The study found expressions for flow parameters such as velocity, resistance to flow, and shear stress. The results compared with the case of normal blood and other models. Ponalgusam [9] presented a study on the effects of slip (at the stenotic wall) and the influence of body acceleration on the flow variables such as velocity profiles, shear stress, flow rate and effective viscosity for pulsatile Newtonian blood flow through a stenosed vessel. Verma [10] has proposed the study of the effect of mild stenosis on the flow of blood represented as a Newtonian fluid, by using the momentum integral techniques to solve the equation governing the flow. The study found analytic expressions for the pressure gradient, velocity profile, shearing stress and separation phenomena at varying Reynolds number.

In this work, we study the two dimensional steady, incompressible Newtonian laminar flow of blood through a stenosed (constricted) artery using the Variational Iteration Method (VIM). Several methods have recently been introduced to solve the governing equations of blood flow such as the homotopy analysis, perturbation analysis, momentum integral techniques, the Galerkin Finite Element method etc. The Variational Iteration Method (as proposed by [11] in 1999 ) is capable of solving the non-linear governing equations of the Newtonian Blood flow.

\section{Flow Geometry}

In this section, we study the axisymmetry steady flow in a stenotic tube. For clarity of purpose, we assumed the blood flow to be laminar, two dimensional, viscous, fully developed, incompressible and Newtonian flow through an artery with a mild stenosis (constriction).

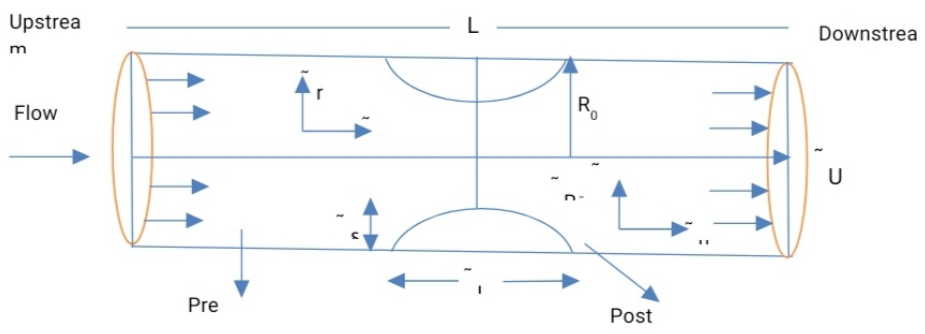

Figure 1. Schematic diagram of an arterial stenosis. 


\section{Flow Analysis and Co-ordinate System}

The constricted wall of this artery is assumed to be rigid and impermeable. The equation of the stenosis surface in figure 1 is mathematically modeled [4] as

$$
\tilde{R}_{(\tilde{Z})}=R_{0}-\frac{\tilde{\delta}}{2}\left(1+\cos \frac{2 \pi}{L_{0}} \tilde{Z}\right)
$$

where,

$\tilde{R}_{(Z)}$ : The radius of the artery in the stenotic region.

$R_{0}$ : The constant radius of the normal artery in the non-stenotic region.

$L$ : The length of the artery.

$\tilde{L_{0}}$ : The length of the stenosis.

$\tilde{Z}$ : The axial coordinate.

$\tilde{\delta}$ : The maximum height of the stenosis.

Note: $(\sim)$ represents dimensional variables which make it different from the corresponding dimensionless variables.

Blood flow has been assumed to be a homogeneous Newtonian fluid and the flow is in two dimensions, in axial and radial directions (coordinates). The incompressible NavierStokes equations along with the continuity equations have been used as the governing equations for modeling the fluid flow.

Since the tube carries blood in horizontal direction, we neglect gravity and the NavierStokes equation for a 2-dimensional steady, incompressible laminar flow takes the form

$$
\tilde{u} \frac{\partial \tilde{u}}{\partial \tilde{z}}+\tilde{v} \frac{\partial \tilde{u}}{\partial \tilde{\Gamma}}=-\frac{1}{\rho}\left(\frac{\partial \tilde{p}}{\partial \tilde{z}}\right)+\frac{\mu}{\rho}\left(\frac{\partial^{2} \tilde{u}}{\partial \tilde{\Gamma}^{2}}+\frac{1}{\Gamma} \frac{\partial \tilde{u}}{\partial \tilde{\Gamma}}+\frac{\partial^{2} \tilde{u}}{\partial \tilde{z}^{2}}\right)
$$

and

$$
\tilde{u} \frac{\partial \tilde{v}}{\partial \tilde{z}}+\tilde{v} \frac{\partial \tilde{v}}{\partial \tilde{\Gamma}}=-\frac{1}{\rho}\left(\frac{\partial \tilde{p}}{\partial \bar{\Gamma}}\right)+\frac{\mu}{\rho}\left(\frac{\partial^{2} \tilde{v}}{\partial \tilde{\Gamma}^{2}}+\frac{1}{\Gamma} \frac{\partial \tilde{v}}{\partial \tilde{\Gamma}}+\frac{\partial^{2} \tilde{v}}{\partial \tilde{z}^{2}}-\frac{\tilde{v}}{\tilde{\Gamma}^{2}}\right)
$$

Where equation (2) and (3) are in the axial and radial directions respectively. Also, for 2-dimensional axisymmetric geometries, the continuity equation is given by

$$
\frac{\partial \tilde{u}}{\partial \tilde{z}}+\frac{\partial \tilde{v}}{\partial \tilde{\Gamma}}+\frac{\tilde{v}}{\tilde{\Gamma}}=0
$$

where, $\tilde{u}$ is the axial velocity, $\tilde{v}$ is the radial velocity, $\tilde{\Gamma}$ is the radial coordinate, $\tilde{z}$ is the axial coordinate, $\rho$ is the density, $\tilde{\rho}$ is the blood pressure and $\mu$ is the viscosity coefficient of blood.

\section{Non-Dimensional Variable Transformation}

We introduce the following non-dimensional variables using the transformations

$$
\tilde{u}=u \tilde{U}_{0}, \tilde{u}=v \tilde{U}_{0}, \tilde{z}=z R_{0}, \tilde{\Gamma}=\Gamma R_{0}, \tilde{p}=p \rho \tilde{U}_{0}^{2}, \tilde{U}=U \tilde{U}_{0}, \tilde{\delta}=\delta R_{0}, \tilde{L}=L_{0} R_{0},
$$


where $\tilde{U}_{0}$ denotes the average velocity in the unobstructed artery and $\tilde{U}$ is the centerline velocity. Therefore, the geometry of the obstruction in equation (1) in dimensionless form becomes

$$
R_{\left(z R_{0}\right)}=R_{0}-\frac{\delta R_{0}}{2}\left(1+\cos \frac{2 \pi}{L_{0}} z R_{0}\right)
$$

This implies that

$$
R_{z}=1-\frac{\delta}{2}\left(1+\cos \frac{2 \pi}{L_{0}} z\right)
$$

Substituting the above non-dimensional variables into equation (2) and multiplying through by $\frac{R_{0}}{\tilde{U}_{0}^{2}}$, we obtain,

$$
\begin{aligned}
u \tilde{U} \frac{\partial u \tilde{U}_{0}}{\partial z R_{0}} \cdot \frac{R_{0}}{\tilde{U}_{0}^{2}}+v \tilde{U} \frac{\partial u \tilde{U}_{0}}{\partial \Gamma R_{0}} \cdot \frac{R_{0}}{\tilde{U}_{0}^{2}}=-\frac{\partial p}{\partial z} \frac{\tilde{U}_{0}^{2}}{R_{0}} \cdot \frac{R_{0}}{\tilde{U}_{0}^{2}}+\frac{\mu}{\rho U_{0}}\left(\frac{\partial^{2} u}{\partial \Gamma^{2} R_{0}}+\frac{1}{\Gamma R_{0}} \frac{\partial u}{\partial \Gamma}+\frac{\partial^{2} u}{\partial z^{2} R_{0}}\right) \\
u \frac{\partial u}{\partial z}+v \frac{\partial u}{\partial \Gamma}=-\frac{\partial p}{\partial z}+\frac{\mu}{\rho U_{0}}\left(\frac{\partial^{2} u}{\partial \Gamma^{2} R_{0}}+\frac{1}{\Gamma R_{0}} \frac{\partial u}{\partial \Gamma}+\frac{\partial^{2} u}{\partial z^{2} R_{0}}\right)
\end{aligned}
$$

For

$$
R_{e}=\frac{2 \rho \tilde{U}_{0} R_{0}}{\mu} \Rightarrow \mu=\frac{2 \rho \tilde{U}_{0} R_{0}}{R_{e}}
$$

Then, $u \frac{\partial u}{\partial z}+v \frac{\partial u}{\partial \Gamma}=-\frac{\partial p}{\partial z}+\frac{2 \rho \tilde{U}_{0} R_{0}}{\rho R_{e} U_{0}}\left(\frac{\partial^{2} u}{\partial \Gamma^{2} R_{0}}+\frac{1}{\Gamma R_{0}} \frac{\partial u}{\partial \Gamma}+\frac{\partial^{2} u}{\partial z^{2} R_{0}}\right)$

This implies that

$$
u \frac{\partial u}{\partial z}+v \frac{\partial u}{\partial \Gamma}=-\frac{\partial p}{\partial z}+\frac{2}{R_{e}}\left(\frac{\partial^{2} u}{\partial \Gamma^{2} R_{0}}+\frac{1}{\Gamma R_{0}} \frac{\partial u}{\partial \Gamma}+\frac{\partial^{2} u}{\partial z^{2} R_{0}}\right)
$$

where $R_{e}=\frac{2 \rho \tilde{U}_{0} R_{0}}{\mu}$ is the Reynolds number. Equation (6) is the Navier-Stoke equation in the axial direction.

Also, substituting the above non-dimensional variables into equation (3) and multiplying through by $\frac{R_{0}}{\tilde{U}_{0}^{2}}$ we obtain,

$$
\begin{aligned}
u \tilde{U}_{0} \frac{\partial u \tilde{U}_{0}}{\partial z R_{0}} \cdot \frac{\tilde{U}_{0}}{R_{0}}+v \tilde{U}_{0} \frac{\partial v \tilde{U}_{0}}{\partial \Gamma R_{0}} \cdot \frac{\tilde{U}_{0}^{2}}{R_{0}} & =-\frac{\partial p \tilde{U}_{0}^{2}}{\partial \Gamma R_{0}} \cdot \frac{\tilde{U}_{0}^{2}}{R_{0}}+\frac{\mu}{\rho} \cdot \frac{\tilde{U}_{0}^{2}}{R_{0}}\left(\frac{\partial^{2} u}{\partial \Gamma^{2} R_{0}}+\frac{1}{\Gamma R_{0}} \frac{\partial u}{\partial \Gamma}+\frac{\partial^{2} u}{\partial z^{2} R_{0}}\right) \\
u \frac{\partial u}{\partial z}+v \frac{\partial u}{\partial \Gamma} & =-\frac{\partial p}{\partial \Gamma}+\frac{2 \rho U_{0} R_{0}}{\rho R_{e} U_{0}}\left(\frac{\partial^{2} v}{\partial \Gamma^{2} R_{0}}+\frac{1}{\Gamma R_{0}} \frac{\partial v}{\partial \Gamma}+\frac{\partial^{2} v}{\partial z^{2} R_{0}-\frac{v U_{0}}{\Gamma^{2} R_{0}}}\right)
\end{aligned}
$$

This implies that

$$
u \frac{\partial v}{\partial z}+v \frac{\partial v}{\partial \Gamma}=-\frac{\partial p}{\partial \Gamma}=\frac{2}{R_{e}}\left(\frac{\partial^{2} v}{\partial \Gamma^{2}}+\frac{1}{\Gamma} \frac{\partial v}{\partial \Gamma}+\frac{\partial^{2} v}{\partial z^{2}-\frac{v}{\Gamma^{2}}}\right)
$$

where $R_{e}=\frac{2 \rho \tilde{U}_{0} R_{0}}{\mu}$ is the Reynolds number. Equation (7) is the Navier-Stoke equation in the radial direction. 
Now, we consider the continuity equation as follows:

Substituting the above non-dimensional variables into equation (4) and multiplying through by $\frac{R_{0}}{\tilde{U}}$, we obtain

$$
\begin{aligned}
& \frac{\partial u \tilde{U}_{0}}{\partial z R_{0}} \cdot \frac{R_{0}}{\tilde{U}_{0}}+\frac{\partial v \tilde{U}_{0}}{\partial \Gamma R_{0}} \cdot \frac{R_{0}}{\tilde{U}_{0}}+v \frac{\tilde{U}_{0}}{\Gamma R_{0}} \cdot \frac{R_{0}}{\tilde{U}_{0}} \\
& \frac{\partial u}{\partial z}+\frac{\partial v}{\partial \Gamma}+\frac{v}{\Gamma}=0
\end{aligned}
$$

\subsection{Boundary Conditions}

The velocity profile constrains for axisymmetric tube flow are given as

i. $u=U$ at $r=0$, is the centre line velocity.

ii. Within the Artery: a "no slip" boundary condition prevails at the walls, i.e, the speed of the blood along the walls vessel is zero, in other words, $u=0$, at $r=R$,

iii. For a finite pressure and inertia forces, as the radius of the element tends to zero, the viscous forces proportional to $\frac{\partial u}{\partial \Gamma}$ tends to zero. That is, $\frac{\partial u}{\partial \Gamma}=0$ at $r=0$.

iv. Eliminating pressure term between (6) and (7) and considering the resulting equations as $r \rightarrow 0$

$\frac{\partial^{3} u}{\partial \Gamma^{3}}$ at $\Gamma=0$.

v. Neglecting the viscous term of the normal stress retarding the flow in the axial direction of equation (6) $\frac{\partial^{2} u}{\partial z^{2}}$, which is an assumption used in the analysis of nonuniform flow and with $\mathrm{u}=\mathrm{v}=0$ at the wall, we have

$$
\frac{\partial p}{\partial z}=\frac{2}{R_{e}} \frac{1}{\Gamma} \frac{\partial}{\partial \Gamma}\left(r \frac{\partial u}{\partial \Gamma}\right), r=R
$$

\section{Method of Solution}

Various numerical methods have been explored in the course of solving the governing equation subject to the prescribed boundary conditions for the problem considered. For example, the Galerkin finite element method was adopted by [12] to solve the considered problem. The variational iteration method (VIM) is a numerical scheme employed here in this research to solve the considered problem. This method was first conceived by a Chinese mathematician [11] in 1999. Since then, the method has been used in seeking approximate solutions to many problems in applied mathematics, see for examples [13-17] and literature cited therein.

The VIM is applied to solve the equations (6), (7) and (8) subject to the boundary conditions in section 4.1. Here, it is assumed that the continuity equation satisfies the velocity profile conditions. The velocity profile, $u$ and the pressure gradients are obtained 
recursively. The VIM converges with high level precision based on the Reynold number, $R_{e}$.

The convergence of solution is obtained when the error recorded for each consecutive iterations is $\left|u_{n+1}-u_{n}\right| \leqslant 10^{-5}, \mathrm{n}$ is the number of iterations of $\mathrm{u}$. Applying the VIM to equation (6) and (7), we construct a correction functional for the axial and radial directions as follows:

$u(n+1)=u_{n}+\int_{0}^{R} \lambda(\Gamma)\left(u_{n} \frac{\partial u_{n}}{\partial z}+v_{n} \frac{\partial u_{n}}{\partial \Gamma}+\frac{\partial p}{\partial z}-\frac{2}{R_{e}}\left(\frac{\partial^{2} u_{n}}{\partial \Gamma^{2}}+\frac{1}{\Gamma} \frac{\partial u_{n}}{\partial \Gamma}+\frac{\partial^{2} u_{n}}{\partial z^{2}}\right)\right) d s, n \geqslant 0$
$v_{(n+1)}=v_{n}+\int_{0}^{R} \lambda(\Gamma)\left(u_{n} \frac{\partial v}{\partial z}+v_{n} \frac{\partial v_{n}}{\partial \Gamma}+\frac{\partial p}{\partial \Gamma}-\frac{2}{R_{e}}\left(\frac{\partial^{2} v_{n}}{\partial \Gamma^{2}}+\frac{1}{\Gamma} \frac{\partial v_{n}}{\partial \Gamma}+\frac{\partial^{2} v_{n}}{\partial z^{2}-\frac{v_{n}}{\Gamma^{2}}}\right)\right) d \Gamma, n \geqslant 0$

where $\lambda(\Gamma)$ is the general Lagrange multiplier, which can be obtained using the generalized formula [18]

$$
\lambda_{n}(\Gamma)=(-1)^{n} \frac{(\Gamma-z)^{(n-1)}}{(n-1) !}
$$

where $n$ is the highest occurring derivative in (11).

Since, the equations (6) and (7) are of second order, we have $\lambda(\Gamma)=(\Gamma-z)$. Therefore, equation (9) becomes

$u_{(n+1)}=u_{n}+\int_{0}^{R}(\Gamma-z)\left(u_{n} \frac{\partial u_{n}}{\partial z}+v_{n} \frac{\partial u_{n}}{\partial \Gamma}+\frac{\partial p}{\partial z}-\frac{2}{R_{e}}\left(\frac{\partial^{2} u_{n}}{\partial \Gamma^{2}}+\frac{1}{\Gamma} \frac{\partial u_{n}}{\partial \Gamma}+\frac{\partial^{2} u_{n}}{\partial z^{2}}\right)\right) d s, n \geqslant 0$

where $v_{n}$ is obtained from the continuity equation, given as;

$$
v_{n}=r\left(\frac{\partial}{\partial z} u_{n}+\frac{\partial}{\partial \Gamma} u_{n}\right)
$$

Equation (12) is used to derive its component parts such as $u_{n}, n \geqslant 0$.

\subsection{Determination of Initial Approximation}

Since our interest is only in the axial flow, we only seek the initial approximation to kick-off the iterative scheme in Equation (12).

Let the initial approximation be given as a power series of the form

$$
u_{0}=U\left[\sum_{1=0}^{n} \alpha_{i} x^{i}\right]
$$

where $x=\left(\frac{\Gamma}{R}\right)$ defines the velocity profile in the axial direction, and $n$ is the degree of the velocity profile polynomials. 
If we choose $n=4$ arbitrarily, then

$$
\begin{aligned}
& u_{0}=\left(\alpha_{0}+\alpha_{1}(x)+\alpha_{2}(x)^{2}+\alpha_{3}(x)^{3}+\alpha_{4}(x)^{4}\right) U \\
& \Rightarrow u_{0}=\left(\alpha_{0}+\alpha_{1}\left(\frac{\Gamma}{R}\right)+\alpha_{2}\left(\frac{\Gamma}{R}\right)^{2}+\alpha_{3}\left(\frac{\Gamma}{R}\right)^{3}+\alpha_{4}\left(\frac{\Gamma}{R}\right)^{4}\right) U
\end{aligned}
$$

Hence, the iterative scheme (VIM) becomes

$$
\begin{aligned}
& u_{0}=\left(\alpha_{0}+\alpha_{1}\left(\frac{\Gamma}{R}\right)+\alpha_{2}\left(\frac{\Gamma}{R}\right)^{2}+\alpha_{3}\left(\frac{\Gamma}{R}\right)^{3}+\alpha_{4}\left(\frac{\Gamma}{R}\right)^{4}\right) U \\
& u_{(n+1)}=u_{n} \int_{0}^{R}\left(u_{n} \frac{\partial u_{n}}{\partial z}+\Gamma\left(\frac{\partial}{\partial z} u_{n}+\frac{\partial}{\partial \Gamma} u_{n}\right) \frac{\partial u_{n}}{\partial \Gamma}+\frac{\partial p}{\partial z}-\frac{2}{R_{e}}\left(\frac{\partial^{2} u_{n}}{\partial \Gamma^{2}}+\frac{1}{\Gamma} \frac{\partial u_{n}}{\partial \Gamma}+\frac{\partial^{2} u_{n}}{\partial z^{2}}\right)\right) d \Gamma, n \geqslant 0
\end{aligned}
$$

Since our interest is axial direction, the flow in radial direction is assumed constants. Hence, we execute equation (15) with the aid of Maple 18 software for $n=0,1,2,3, \cdots$. Thus for every value of $n \geqslant 0$, we obtain $u_{n+1}=u_{n}$. That is,

$$
u_{0}=u_{1}=u_{2}=\cdots=\left(\alpha_{0}+\alpha_{1}\left(\frac{\Gamma}{R}\right)+\alpha_{2}\left(\frac{\Gamma}{R}\right)^{2}+\alpha_{3}\left(\frac{\Gamma}{R}\right)^{3}+\alpha_{4}\left(\frac{\Gamma}{R}\right)^{4}\right) U
$$

This implies our approximate solution for $n \geqslant 0$ is the velocity profile polynomial of the fourth-degree, that is,

$$
\frac{u}{U}=\alpha_{0}+\alpha_{1}\left(\frac{\Gamma}{R}\right)+\alpha_{2}\left(\frac{\Gamma}{R}\right)^{2}+\alpha_{3}\left(\frac{\Gamma}{R}\right)^{3}+\alpha_{4}\left(\frac{\Gamma}{R}\right)^{4}
$$

Now, applying the prescribed boundary conditions to equation (17) to get $\alpha_{i}, i=0,1,2,3,4$. At the boundary $u=U, \Gamma=0$, we have $\alpha_{0}=1$.

At the boundary $u=0, \Gamma=R$, we have

$$
1+\alpha_{1}+\alpha_{2}+\alpha_{3}+\alpha_{4}=0
$$

At the boundary $\frac{\partial u}{\partial \Gamma}=0, \Gamma=0$, we have $\alpha_{1}=0$.

At the boundary $\frac{\partial^{2} u}{\partial \Gamma^{2}}=0, r=0$, we have

$\frac{\partial^{3} u}{\partial \Gamma^{3}}=\frac{6 \alpha_{3}}{R^{3}}+\frac{24 \Gamma \alpha_{4}}{R^{4}}=0$.

But $r=0 \Rightarrow \frac{6 \alpha_{3}}{R^{3}}=0 \Rightarrow \alpha_{3}=0$.

Since, $\alpha_{0}=1, \alpha_{1}=0, \alpha_{3}=0$, then we have from equation (17),

$$
\alpha_{2}=-1-\alpha_{4}
$$


At the boundary $\frac{\partial p}{\partial z}=\frac{2}{R_{e}} \frac{1}{\Gamma} \frac{\partial}{\partial \Gamma}\left(\Gamma \frac{\partial u}{\partial \Gamma}\right), \Gamma=R$, we have

$$
\begin{aligned}
& \frac{R_{e} \Gamma \partial p}{2 \partial z}=\frac{\partial}{\partial \Gamma}\left(\Gamma \frac{\partial u}{\partial \Gamma}\right)=\Gamma \frac{\partial^{2} u}{\partial \Gamma^{2}}+\Gamma \frac{\partial u}{\partial \Gamma}, r=R \\
& \Rightarrow \frac{R_{e} R \Gamma \partial p}{2 \partial z}=\frac{\partial}{\partial \Gamma}\left(\Gamma \frac{\partial u}{\partial \Gamma}\right)=R\left(\frac{2 U \alpha_{2}}{R^{2}}+\frac{12 \alpha_{4}}{R^{2}}+\frac{2 U \alpha_{2}}{R}+\frac{4 U \alpha_{2}}{R}\right) \\
& \Rightarrow \frac{R_{e} R \Gamma \partial p}{2 \partial z}=\frac{2 U \alpha_{2}+12 \alpha_{4}+2 U \alpha_{2}+4 U \alpha_{4}}{R} \\
& \Rightarrow \frac{R_{e} R^{2} \Gamma \partial p}{2 U \partial z}=2 \alpha_{2}+16 \alpha_{4} \\
& \Rightarrow \frac{R_{e} R^{2} \Gamma \partial p}{8 U \partial z}=\alpha_{2}+4 \alpha_{4}
\end{aligned}
$$

Since, $\alpha_{2}=-1-\alpha_{4}$, equation (20) becomes

$$
\frac{R_{e} R^{2} \Gamma \partial p}{8 U \partial z}+1=3 \alpha_{4}
$$

From equation (21), we have that

$$
\alpha_{4}=\frac{\frac{R_{e} \Gamma R^{2} \partial p}{8 U \partial z}+1}{3}
$$

Similarly,

$$
\alpha_{2}=-\frac{4}{3}-\frac{\frac{R_{e} R^{2} \Gamma \partial p}{8 U \partial z}}{3}
$$

Substituting $\alpha_{n}, n=0(1) 4$, into equation (17), we obtain

$$
\frac{u}{U}=1-\frac{4}{3}-\frac{\frac{R_{e} R^{2} \Gamma \partial p}{8 U \partial z}}{3}\left(\frac{\Gamma}{R}\right)^{2}+\frac{\frac{R_{e} R^{2} \Gamma \partial p}{8 U \partial z}+1}{3}\left(\frac{\Gamma}{R}\right)^{4} .
$$

If we let

$$
\Psi=\frac{\frac{R_{e} \Gamma R^{2} \partial p}{8 U \partial z}}{3}
$$

then equation (24) can be written as

$$
\frac{u}{U}=1-\frac{4}{3}-\frac{\Psi}{3}\left(\frac{\Gamma}{R}\right)^{2}+\frac{(-\Psi-1)}{3}\left(\frac{\Gamma}{R}\right)^{4} .
$$

Evidently, if $\Psi=1[12]$, we have a parabolic velocity profile which corresponds to the Poiseuille profile

$$
\frac{u}{U}=1-\left(\frac{\Gamma}{R}\right)^{2}
$$




\subsection{The Flow Flux}

The flow flux denoted by $\mathrm{Q}$ is defined as [12]

$$
Q=2 \pi \int_{0}^{R} \Gamma u d \Gamma
$$

From equation (25), we have

$$
u=U\left(1-\frac{4}{3}-\frac{\Psi}{3}\left(\frac{\Gamma}{R}\right)^{2}+\frac{(-\Psi-1)}{3}\left(\frac{\Gamma}{R}\right)^{4}\right)
$$

Thus, equation (27) can be written as

$$
\begin{gathered}
Q=2 \pi \int_{0}^{R} \Gamma\left(1-\frac{4}{3}-\frac{\Psi}{3}\left(\frac{\Gamma}{R}\right)^{2}+\frac{(-\Psi-1)}{3}\left(\frac{\Gamma}{R}\right)^{4}\right) d \Gamma \\
Q=2 \pi U\left[\frac{R^{2}}{2}-\frac{1}{12}(4-\Psi) R^{2}+\frac{1}{18}(1-\Psi) R^{2}\right] \\
\Rightarrow Q=2 \pi U R^{2}\left[1-\frac{1}{12}(4-\Psi)+\frac{1}{18}(1-\Psi)\right] \\
\Rightarrow Q=\frac{1}{18} \pi U R^{2}[(8+\Psi)]
\end{gathered}
$$

Since $\Psi=-\frac{R_{e} R^{2} \Gamma \partial p}{8 U \partial z}$, equation (29) becomes

$$
Q=\frac{1}{18} \pi U R^{2}\left[8-\frac{\left(R_{e} R^{2} \partial p\right)}{8 U \partial z}\right]=\frac{4}{9} \pi U R^{2}-\frac{\left(\pi R_{e} R^{4} \partial p\right)}{144 \partial z}
$$

From equation (26), we have that

$$
U=\frac{9}{4} \frac{1}{R^{2}}\left[Q+\frac{\left(\pi R_{e} R^{4} \partial p\right)}{144 \partial z}\right]
$$

Equation (31) is called the centerline velocity.

Now, using $U=\frac{9}{4} \frac{1}{R^{2}}\left[Q+\frac{\left(\pi R_{e} R^{4} \partial p\right)}{144} \frac{\partial p}{\partial z}\right]$ in equation (25), we have,

$$
u=\frac{9}{4} \frac{1}{R^{2}}\left[Q+\frac{\left(\pi R_{e} R^{4}\right)}{144} \frac{\partial p}{\partial z}\right]\left[1-\frac{4}{3}-\frac{\Psi}{3}\left(\frac{\Gamma}{R}\right)^{2}+\frac{(-\Psi-1)}{3}\left(\frac{\Gamma}{R}\right)^{4}\right]
$$

But $\Psi=-\frac{R_{e} R^{2} \Gamma \partial p}{8 U \partial z}$,

then equation (32) becomes

$$
u=\frac{9}{4} \frac{1}{R^{2}}\left[Q+\frac{\left(\pi R_{e} R^{4}\right)}{144} \frac{\partial p}{\partial z}\right]\left[1-\frac{4}{3}-\frac{1}{3}\left(-\frac{R_{e} R^{2} \Gamma \partial p}{8 U \partial z}\right)\left(\frac{\Gamma}{R}\right)^{2}+\frac{1}{3}\left(\frac{R_{e} R^{2} \Gamma \partial p}{8 U \partial z}+1\right)\left(\frac{\Gamma}{R}\right)^{4}\right]
$$


Equation (33) is the velocity profile equation.

Similarly, substituting (26) and (31) in $\frac{\partial p}{\partial z}=\frac{2}{R_{e}} \frac{1}{\Gamma} \frac{\partial}{\partial \Gamma}\left(\Gamma \frac{\partial u}{\partial \Gamma}\right), \Gamma \geqslant R$, we have

$$
\begin{gathered}
\frac{\partial p}{\partial z}=\frac{2}{R_{e}} \frac{1}{\Gamma}\left(R \frac{\partial^{2} u}{\partial \Gamma^{2}}+\frac{\partial u}{\partial \Gamma}\right) \\
u=U\left(1-\left(\frac{\Gamma}{R}\right)^{2}\right)=\frac{9}{4} \frac{1}{R^{2}}\left[Q+\frac{\left(\pi R_{e} R^{4} \partial p\right)}{144 \partial z}\right]\left[1-\left(\frac{\Gamma}{R}\right)^{2}\right]
\end{gathered}
$$

Evaluating $\frac{\partial u}{\partial \Gamma}$ and $\frac{\partial^{2} u}{\partial \Gamma^{2}}$ using equation (35) with the aid of Maple 18 software at, $\mathrm{r}=\mathrm{R}$, and substituting in equation (33) yield the result,

$$
\frac{\partial p}{\partial z}=\frac{39}{9} \frac{Q^{2}}{\pi^{2}} \frac{1}{R^{5}} \frac{d R}{d z}-\frac{16}{\pi} \frac{Q}{R_{e} R^{4}}
$$

Equation (36) is called the pressure gradient and $\left|\frac{\partial p}{\partial z}\right|$ is the absolute pressure gradient.

\subsection{Results and Discussion}

In order to demonstrate the applicability of the mathematical model derived and analyzed in the preceding sections, expressions for pressure and velocity profile (axial direction) are conditioned in the no velocity-slip condition at the arterial wall. For us to have a vivid insight of the axisymmetric steady flow of blood in an embolic table, the variations of $u, \Psi, Q$ and $\frac{\partial p}{\partial x}$ have been derived and the solutions are presented graphically with the aid of the computer application software Maple 18. The axisymmetric steady flow of blood is modeled as Newtonian and incompressible through an artery with a fixed solid stenosis. The variation iteration method was used to solve the governing equations with the assumption that the initial approximation is defined as a polynomial of the velocity profile of fourth-degree. Implementing the VIM on equation (17) for $n \geqslant 0$ with Maple 18 software, we observe that $u_{0}=u_{1}=u_{2}=\cdots=u$. In our analysis, the maximum height of the stenosis is taken as 0.25 or $\frac{1}{4}$. However, the maximum height of the stenosis is defined in the range 0 to 0.6 to enable us analyze the effect pronounce. The absolute pressure gradient parameter, $\left|\frac{\partial p}{\partial z}\right|$ is defined in the range in 0 to 1 . The velocity slip parameter is defined in the range 0 to 0.6 . In this work, the Reynold number is taken in the range 20, 60 and 100 for $\delta=\frac{1}{4}$, to enable us analyze the effect pronounce.

The Figures 2-3 illustrate the axial velocity profile for different values of Reynold numbers for $\delta=\frac{1}{4}$ in the analysis of axisymmetric steady flow of blood in an embolus tube. It is observed in figure 1 that the no velocity slip at the walls of the arteries perturbs the velocity due to the obstructed arterial segments of the tube. It is also observed at the throat of the stenosis $(u=1.6)$, the axial velocity increases with $\mathrm{R}=0.1$ and 0.2 with increase in the radial distance. However, for $R=0.3$ and 0.4 , the trend is metamorphosed. Similarly, at $u=1.8$ and 1.9 (the throat of the stenosis) the axial velocity profile drastically increases as shown in the Figures 3 and 4. Figures 5-7 illustrate the absolute values of the dimensionless pressure gradient $\left|\frac{\partial p}{\partial x}\right|$ defined in the range 0 to 1 for $\delta=\frac{1}{4}$ for the Reynold 
numbers 20, 30 and 50. It is observed that the pressure gradient is at the throat of stenosis as the Reynold number increases. However, the variation of the pressure gradient is mostly felt at the downstream of the tube.

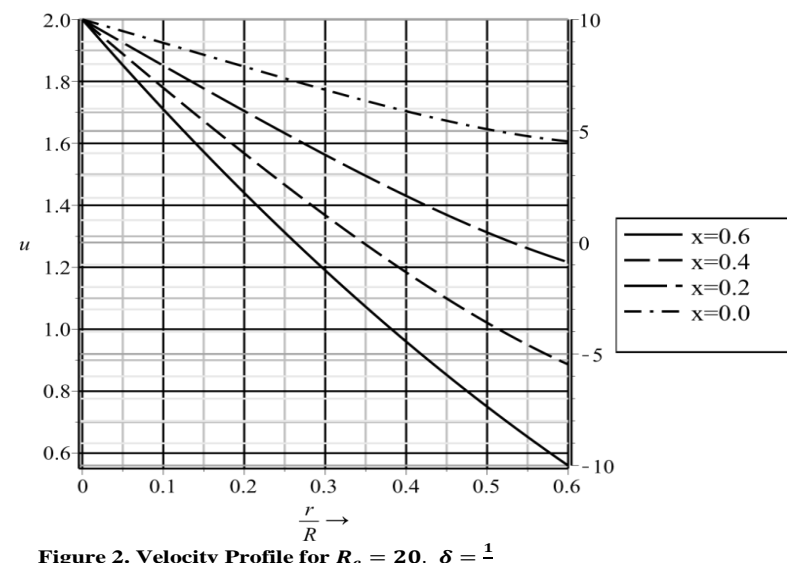

Figure 2. Velocity Profile for $R_{e}=20, \delta=\frac{1}{4}$

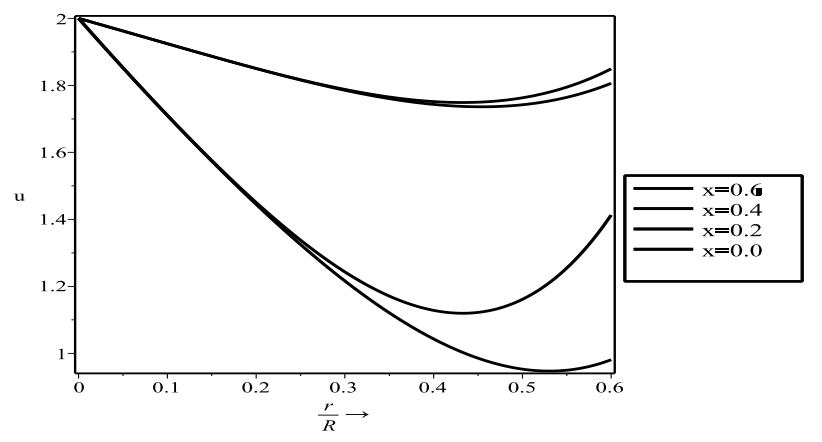

Figure 4. Velocity Profile for $R_{e}=1000, \delta=\frac{1}{4}$

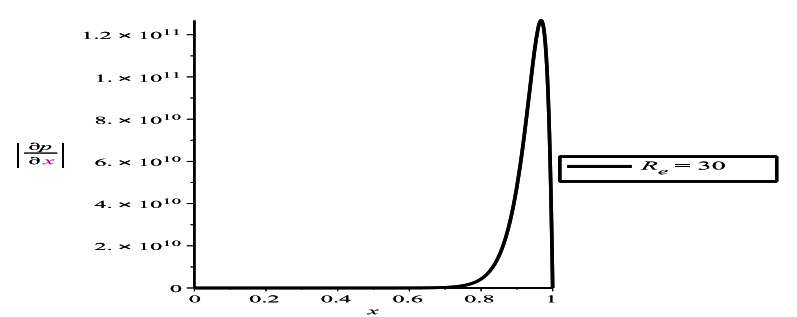

Figure 6. Absolute pressure gradient for $\delta=\frac{1}{4}$.

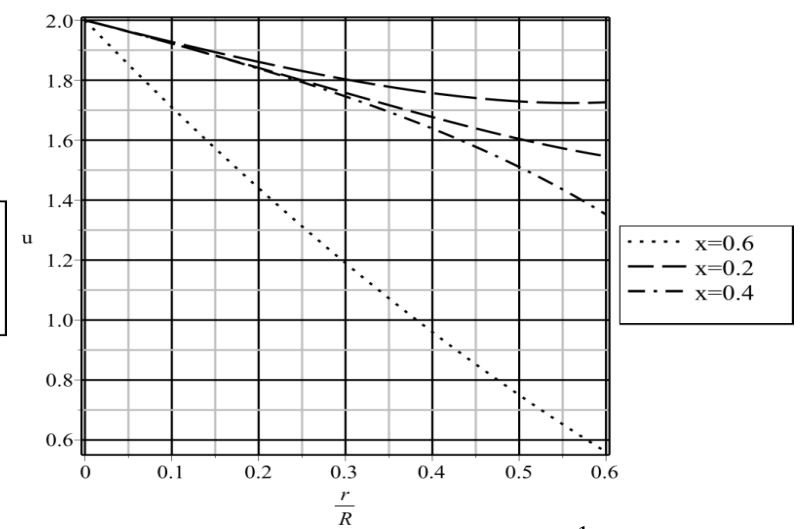

Figure 3. Velocity Profile for $R_{e}=60, \delta=\frac{1}{4}$

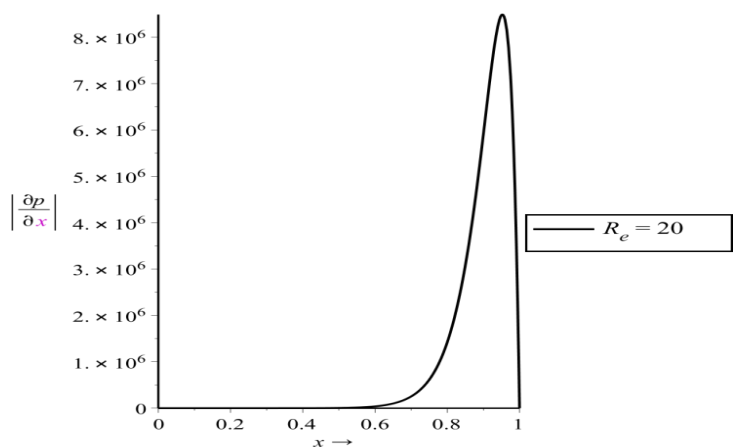

Figure 5. Absolute pressure gradient for $\delta=\frac{1}{4}$.

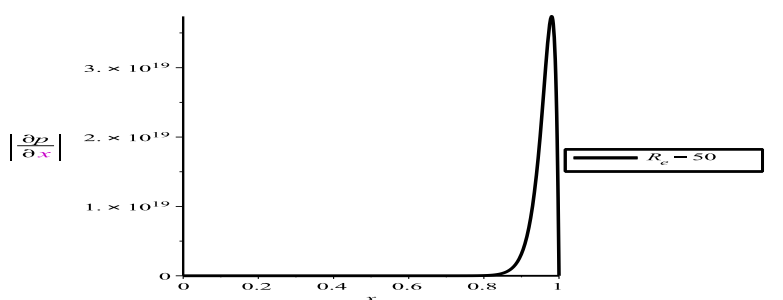

Figure 7. Absolute pressure gradient for $\delta=\frac{1}{4}$. 


\section{Conclusion}

In this research work, we presented the numerical approach for modeling the axisymmetric flow of blood in an embolus tube involving two dimensional Navier-Stoke equations. The VIM coincides with the integral momentum equation method [12], which yields the same sets of equations for the velocity profile and pressure gradient equations. Results from this model corresponds with that of the most resent experimental data that explain how the effect of obstruction to the flow of blood in the artery of the human body with reference to pressure and velocity of the blood flow pass an embolus.

\section{References}

[1] Mark, R. G. (2013). Physiological Fluid dynamics. Department of Electrical Engineering, Mechanical Engineering and the Harvard-MIT Division of Health Sciences and Technology.

[2] Chien, S. (1981). Hemorheology in Clinical Medicine, Recent Advances in Cardiovascular Diseases, 2:21 -26.

[3] Yamaguchi, T. (1996). Advances in Hemodynamics and Hemorheology. 1:201-227.

[4] Young, D. F. (1968). Effect of a Time-Dependent Stenosis On Flow Through a Tube. Trans. ASME, J. Energy Ind. 90: 248-254. http ://books.google.com.ng/books/about/onaortic_Blood_Flow_Simulations.html?id $=J 3 e 9 D A E A C A A J \& r e d i r \_e s c=y$. web11th July, 2017.

[5] Morgan, B. E. and Young,D.F. (1974). An Integral Method for Analysis of Flow in Arterial Stenosis. Bull. Math. Biol. 36: 39-53.

[6] Lee, B. Y., Assadi, C., Madden, J. L. et al. World, J. Surg. (1978). Hemodynamics of Arterial Stenosis. World Journal of Surgery. 2(5): 621-627.

[7] Kapur, J. N. (1984). Mathematical Models in Medical Sciences. International Journal of mathematical education in science and Technology. 5(5):587-600.

[8] Pralhad, R. N. and Schultz, D. H.(2004). Modeling of Arterial Stenosis and Its Applications to Blood Diseases. Mathematical Biosciences 190(2004), 203-220.

[9] Ponalgusam, R. (2007). Blood Flow Through an Artery with Mild Stenosis: A TwoLayered Model, Different Shapes of Stenosis and Slip Velocity at the Wall. Journal of Applied Sciences, 14: 219-238.

[10] Verma, S. R. (2008). Study of Blood Flow Through Modeled Vascular Stenosis. Department of mathematics. D.A-V(P.G). college, Kanpur 208001, India. 
[11] He, J. H. (1999). Variational Iteration Method -A kind of Non-linear Analytic Technique: Some Examples. International Journal of Non-linear Mechanics. Vol. 34, issue 4 699-708.

[12] Mohammed, M. H. (2015). Study of Fluid Flow Through a Tube with and Without Obstacle. Department of mathematics, Bangladesh University of Engineering and Technology. Dhaka-1000, Bangladesh. pp.18-35.

[13] Saberi-Nadjafi, J. and Tamamar, M. (2008). The Variational Iteration Method. A highly promising method for solving the system of integro-differential equations. Computers and Mathematics with Applications. 56:346-351.

[14] Mamadu, E. J. and Tsetimi, J. (2017). Variational Iteration Method for Solving SchroDinger Equations Using Adomian Polynomials. Journal of the Nigerian Association of Mathematical Physics, Vol. 40, pp 11-14.

[15] Tsetimi, J. and Mamadu, E. J. (2017). Solutions of the Fifth Order Boundary Value Problem Using Variational Iteration Decomposition Method and Variational Iteration Method with Chebychev Polynomials. Journal of the Nigerian Association of Mathematical Physics, Vol. 40, pp 39-42.

[16] Abbasbandy, S. and Shivanian, E. (2008).Application of The Variational Iteration Method for System of Nonlinear Volterra's Integro-Differential Equations. Mathematics and Computational Applications, 14(2):147-158.

[17] Tsetimi, J. and Mamadu, E. J. (2021). A New Numerical Approach for Solution to Cauchy Partial Differential Equation Using Mamadu-Njoseh Polynomials. Ciencia e Technica Vitivinicola. Vol. 36(2). pp. 1-8. 